\title{
Believing in seeing
}

\author{
Materials-based imaging agents are attractive candidates for a diverse range of imaging modalities \\ and combined imaging-therapy applications, but economic implications and practical concerns remain \\ obstacles to their clinical translation.
}

Medicine is becoming more and more of a science. And, like any other scientific discipline, probing the problem or, in this case, the patient is the first step in what is often a long process. It's the clinician's hope that such investigations using noninvasive imaging techniques will lead to an accurate diagnosis and, if necessary, a much better informed situation for drawing up an appropriate treatment plan. A myriad of imaging methods most prominently, magnetic resonance imaging, optical imaging, ultrasonography, positron emission tomography and X-ray computed tomography - have become established over the past few decades. In turn, the range of small molecules, molecular complexes and micro- and nanoscale materials used as tracers, targeting or contrast agents has undergone considerable diversification. In this issue, we highlight recent advances in materials-based imaging agents and offer an outlook of the prospects of bioimaging as an essential tool to progress the medical sciences.

Imaging techniques have their own strengths and weaknesses - in terms of resolution, sensitivity and penetration depth - and it is tackling this variation in their application that has enabled materials science to come into its own within the field of bioimaging. More specifically, nanomaterials that can encapsulate or be functionalized with small molecules (for example fluorophores or radionuclides) have allowed the combination of several imaging techniques into one nanoscale platform together with the possibility of adding drug delivery and/or photothermal therapy capabilities. Such potential for multifunctionality also brings conceptual, technical and practical challenges for researchers attempting to achieve success with the design and demonstration in the clinic of materials-based imaging agents. It requires the collaboration of scientists from a range of different disciplines who are able to design and fabricate agents, are knowledgeable about imaging techniques, and have an in-depth understanding of biochemistry and pharmacology as well as disease pathology.

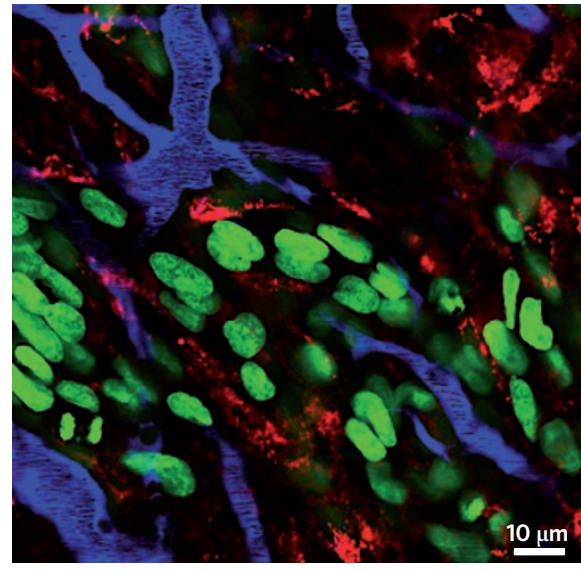

Tumour-associated macrophages (red) can be detected within subcutaneously grown tumours (green) using multimodal nanoparticles. Vessels are stained blue ${ }^{3}$

Cancer imaging is a major player when it comes to bioimaging. In a Commentary on page 110 of this issue, Chun Li highlights recent advances in molecular imaging and theranostics within oncology, and stresses the importance of a high signalto-background ratio for bioimaging agents ${ }^{1}$. Coincidently, Jinming Gao and colleagues report on page 204 a polymeric $\mathrm{pH}$-sensitive nanoprobe that remains silent during circulation in the blood yet is activated within the acidic $\mathrm{pH}$ environment of the tumour ${ }^{2}$. In their Review Article, Ralph Weissleder and colleagues also discuss cancer imaging in relation to macrophage imaging - and extend their discussion into other disease areas that involve macrophage uptake and activity, namely, atherosclerosis, myocardial infarction, aortic aneurysm and diabetes ${ }^{3}$. Macrophage imaging incorporates an impressively wide range of different materials including magnetic, fluorescent, silica and polymeric nanoparticles, in addition to quantum dots and upconversion nanoparticles.

In a Commentary on page 106, Joseph $\mathrm{Wu}$ and colleagues discuss molecular imaging techniques that can be applied to analyse and determine gene- and cell-fate in vivo ${ }^{4}$. The safety and regulatory concerns associated with gene therapy may be more rapidly addressed if accurate information of cellular and graft behaviour could be gathered using imaging techniques.

Leaving the science aside, there are economic issues that cannot be ignored when considering the role of bioimaging in research and medicine. The development of materials as solely imaging agents is less lucrative than launching drug-delivery agents or new therapies, as Chun Li notes in his Commentary. For investment to be realized, there must be a clear advantage to using the material and a much reduced safety concern than that associated with the use of nanoparticles for drug delivery, or their application may become rapidly unjustified. Moreover, the material must produce reliable results, or clinicians carrying out imaging scans on a regular basis are likely to discard them - a potential problem for systems that are polydisperse in nature. Also, accessibility to expensive machines and the availability of trained personnel to perform scans, procedures and maintenance has the potential to make the reality of bioimaging unattainable in the developing world, as well as in developed countries where cost plays a large part of the decision-making within a diagnosis or treatment plan.

Finally, it's important to remember that medicine is not an exact science. For example, screening for some cancers is known to be a far from ideal way of determining the individuals that require treatment. Cancers can lie dormant in people for many years, and over-analysis may result in unnecessary exposure of individuals to radiation and the side effects of imaging agents, and even result in needless surgical procedures. After all, the information obtained from imaging techniques is only one piece of the diagnostic jigsaw. It is the patient and their symptoms that must be considered and treated, rather than just believing the images we see.

\section{References}

1. Li, C. Nature. Mater. 13, 110-115 (2014).

2. Wang, Y. et al. Nature Mater. 13, 204-212 (2014).

3. Weissleder, R., Nahrendorf, M. \& Pittet, M. Nature. Mater 13, 125-138 (2014)

4. Kooreman, N. G., Ransohoff, J. D. \& Wu, J. C. Nature Mater. 13, 106-109 (2014). 